PROCEEDINGS OF THE

AMERICAN MATHEMATICAL SOCIETY

Volume 128, Number 8, Pages 2487-2491

S 0002-9939(00)05615-X

Article electronically published on February 25, 2000

\title{
A CONTINUOUS DECOMPOSITION OF THE MENGER CURVE INTO PSEUDO-ARCS
}

\author{
JANUSZ R. PRAJS
}

(Communicated by Alan Dow)

\begin{abstract}
It is proved that the Menger universal curve $\mathcal{M}$ admits a continuous decomposition into pseudo-arcs with the quotient space homeomorphic to $\mathcal{M}$.
\end{abstract}

Wilson proved [8] Anderson's announcement [1] saying that for any Peano continuum $X$ the Menger universal curve $\mathcal{M}$ admits a continuous decomposition into homeomorphic copies of $\mathcal{M}$ such that the quotient space is homeomorphic to $X$. Anderson also announced (unpublished) that the plane admits a continuous decomposition into pseudo-arcs. This result was proved by Lewis and Walsh [4].

In a previous paper [6] the author has proved that each locally planar Peano continuum with no local separating point admits a continuous decomposition into pseudo-arcs. Applying this result, we prove in this note that the Menger universal curve $\mathcal{M}$ also admits such a decomposition. We can topologically obtain $\mathcal{M}$ and some other continua as the quotient space, but not all Peano continua.

\section{General CONSTRUCTIONS AND THEIR PROPERTIES}

For any compact metric space $X$ let $\Psi_{\omega}(X)$ be the set of all sequences $\left\{X_{n}\right\}$, for $n \in \mathcal{N}=\{\infty, \in, \ldots\}$, of closed, mutually disjoint, nonempty subsets of $X$. Next, let $C$ be the standard Cantor set in the unit interval [0,1]. Fix a sequence of open intervals $\left(a_{n}, b_{n}\right)$ composed of all, mutually different components of $[0,1]-C$. Given a compactum $X$ and a sequence $\left\{X_{n}\right\} \in \Psi_{\omega}(X)$, in the product $C \times X$ identify all pairs of points $\left\langle a_{n}, x\right\rangle$ and $\left\langle b_{n}, x\right\rangle$, where $x \in X_{n}$ and $n \in \mathcal{N}$. Observe that this identification yields an upper semi-continuous decomposition of $C \times X$. Denote by $Q\left(X,\left\{X_{n}\right\}\right)$ the quotient space of this decomposition and by $q$ the quotient mapping.

Property 1. For any compactum $X$ and any sequence $\left\{X_{n}\right\} \in \Psi_{\omega}(X)$, we have $\operatorname{dim} X=\operatorname{dim} Q\left(X,\left\{X_{n}\right\}\right)$.

Proof. Letting $Q=Q\left(X,\left\{X_{n}\right\}\right)$, observe that $Q$ contains copies of $X$, and thus $\operatorname{dim} Q \geq \operatorname{dim} X$.

Fix any positive integer $n$, take the permutation $\left\{i_{1}, \ldots, i_{n}\right\}$ of $\{1, \ldots, n\}$ satisfying $0<a_{i_{1}}<b_{i_{1}}<\ldots<a_{i_{n}}<b_{i_{n}}<1$, and define $I_{0}=\left[0, a_{i_{1}}\right], I_{k}=\left[b_{i_{k}}, a_{i_{k+1}}\right], I_{n}=$

Received by the editors July 2, 1997 and, in revised form, September 11, 1998.

2000 Mathematics Subject Classification. Primary 54F15, 54F50.

Key words and phrases. Continuous decomposition, local separating point, Menger curve, pseudo-arc, quotient space. 
$\left[b_{i_{n}}, 1\right]$ for $k \in\{1, \ldots, n-1\}$. Consider the equivalence in $Q$ identifying all pairs of points $q\left(\left\langle c_{1}, x\right\rangle\right), q\left(\left\langle c_{2}, x\right\rangle\right)$, such that

either $c_{1}, c_{2} \in I_{k}$ for some $k \in\{0, \ldots, n\}$, or

$c_{1}, c_{2} \in I_{k} \cup I_{k+1}$ and $x \in X_{i_{k+1}}$ for some $k \in\{0, \ldots, n-1\}$.

Evidently, this equivalence yields an upper semi-continuous decomposition of $Q$, so denote by $f_{n}: Q \rightarrow f_{n}(Q)$ the quotient mapping. We see that

$$
f_{n}(Q)=f_{n}\left(q\left(\left\{a_{1}\right\} \times X\right)\right) \cup \ldots \cup f_{n}\left(q\left(\left\{a_{n}\right\} \times X\right)\right) \cup f_{n}(q(\{1\} \times X)) .
$$

Thus $f_{n}(Q)$ is composed of the union of $n+1$ topological copies of $X$. Therefore $\operatorname{dim} f_{n}(Q)=\operatorname{dim} X$. Since we have

$$
\lim _{n} \max \left\{\operatorname{diam} f_{n}^{-1}(z): z \in f_{n}(Q)\right\}=0,
$$

then $\operatorname{dim} Q \leq \operatorname{dim} X$.

An easy proof of the following property is left to the reader.

Property 2. If $X$ is a continuum and $\left\{X_{n}\right\} \in \Psi_{\omega}(X)$, then $Q\left(X ;\left\{X_{n}\right\}\right)$ is a continuum.

If, additionally, a point $y=q(\langle c, x\rangle)$ separates the continuum $Q\left(X ;\left\{X_{n}\right\}\right)$, then $c \in\left\{a_{n}, b_{n}\right\}$ and $X_{n}=\{x\}$ for some $n$.

Let $X$ be a compactum, $\left\{X_{n}\right\} \in \Psi_{\omega}(X)$ and let $y=q(\langle c, x\rangle)$ be a point of $Q=$ $Q\left(X ;\left\{X_{n}\right\}\right)$. Take any closed neighborhood $K$ of $x$ in $X$, and positive integers $i, j$ such that $b_{i}<a_{j}$ and $q^{-1}(y) \subset\left(C \cap\left[b_{i}, a_{j}\right]\right) \times K$. Let $n_{k}$ be a sequence of all positive integers such that $\left[a_{n_{k}}, b_{n_{k}}\right] \subset\left[b_{i}, a_{j}\right]$. Notice that the set $L=q\left(\left(C \cap\left[b_{i}, a_{j}\right]\right) \times K\right)$ is a closed neighborhood of $y$ in $Q$, and the family of all such sets $L$ form a basis of closed neighborhoods of $y$ in $Q$.

Assuming that $X$ is a locally connected continuum, we can take $K$ to be a continuum.

If, moreover, $X_{n}$ converges to $X$ in the sense of the Hausdorff distance, then $X_{n_{k}} \cap K \neq \emptyset$ for almost all $k$. If this intersection is empty for some $k$ 's, we modify interval $\left[b_{i}, a_{j}\right]$ to some interval $\left[b_{l}, a_{m}\right] \subset\left[b_{i}, a_{j}\right]$ so that we have again $q^{-1}(y) \subset\left(C \cap\left[b_{l}, a_{m}\right]\right) \times K$, and $\left(a_{n_{k}}, b_{n_{k}}\right) \cap\left[b_{l}, a_{m}\right]=\emptyset$ for those $k$ 's. Then for each $r$ such that $\left[a_{r}, b_{r}\right] \subset\left[b_{l}, a_{m}\right]$ we have $X_{r} \cap K \neq \emptyset$. Therefore the neighborhood $J=q\left(\left(C \cap\left[b_{l}, a_{m}\right]\right) \times K\right)$ of $y$ in $Q$ is connected. Indeed, $J$ is homeomorphic to a space of the form $Q\left(K ;\left\{X_{r_{k}} \cap K\right\}\right)$, where $r_{k}$ is a sequence of all positive integers $r$ from the previous statement, so it is connected by Property 2

If, additionally, each $X_{n}$ is dense in itself, then $\{x\} \neq X_{r_{k}} \cap K$ for any $k$. Hence $y$ cannot separate $J$ by Property 2 ,

We have proved the following property.

Property 3. If $X$ is a locally connected continuum and a sequence $\left\{X_{n}\right\} \in \Psi_{\omega}(X)$ converges to $X$ in the sense of the Hausdorff distance, then $Q\left(X,\left\{X_{n}\right\}\right)$ is a locally connected continuum.

If, additionally, each $X_{n}$ is dense in itself, then $Q\left(X,\left\{X_{n}\right\}\right)$ has no local separating point.

In the next proposition we provide a construction of mappings between the spaces of type $Q\left(X ;\left\{X_{n}\right\}\right)$. The proof of this proposition is easy and natural, so we omit it. 
Proposition 4. Let $f: X \rightarrow Y$ be a continuous mapping between compacta $X$ and $Y$, and let $\left\{Y_{n}\right\} \in \Psi_{\omega}(Y)$. Then there is the unique mapping $g: Q\left(X ;\left\{f^{-1}\left(Y_{n}\right)\right\}\right) \rightarrow$ $Q\left(Y ;\left\{Y_{n}\right\}\right)$ such that (for $q_{1}, q_{2}$ being the respective natural quotient mappings) the diagram

$$
\begin{array}{ccc}
C \times X & \stackrel{\text { idc }_{\mathrm{C}}}{\longrightarrow} & C \times Y \\
\downarrow^{q_{1}} & & \downarrow^{q_{2}} \\
Q\left(X ;\left\{f^{-1}\left(Y_{n}\right)\right\}\right) & \stackrel{g}{\longrightarrow} & Q\left(Y ;\left\{Y_{n}\right\}\right)
\end{array}
$$

commutes, and this mapping is continuous.

Moreover, if $f$ is open (surjective), then $g$ is open (surjective) too.

Remark 5. Note that for the above mapping $g$ and for any $p=q_{2}(\langle c, y\rangle)$ in $Q\left(Y ;\left\{Y_{n}\right\}\right)$ fiber $g^{-1}(p)$ is homeomorphic to fiber $f^{-1}(y)$.

Remark 6. Observe that, actually, the pattern of the Lebesgue mapping from the Cantor set $C$ to an arc (identifying each pair $a_{n}, b_{n}$ to a point) was employed in the construction of the spaces $Q\left(X ;\left\{X_{n}\right\}\right)$. Take any continuous surjection $m: C \rightarrow F$ such that

(i) $F$ is a locally connected curve;

(ii) $F$ contains a countable set $F_{0}$ and admits a basis $\left\{B_{1}, B_{2}, \ldots\right\}$ such that bdB is a finite subset of $F_{0}$ for each $i$; and

(iii) if $m^{-1}(p)$ is nondegenerate, then $p \in F_{0}$ for each $p \in F$.

For any compactum $X$ and any sequence $\left\{X_{n}\right\} \in \Psi_{\omega}(X)$ we can obtain a space $Q_{m}\left(X ;\left\{X_{n}\right\}\right)$ analogous to the space $Q\left(X ;\left\{X_{n}\right\}\right)$, where mapping $m$ plays the role of the Lebesgue mapping. These new spaces have properties similar to Properties 1 20 and to Proposition 4. Here we do not develop this generalization, for spaces $Q\left(X ;\left\{X_{n}\right\}\right)$ are sufficient for our purposes.

\section{Decompositions of the Menger Curve}

First, we use the results of the previous section to obtain the following construction of topological Menger curves.

Proposition 7. Let $X$ be a locally connected curve containing no free arc. Then for any sequence $\left\{X_{n}\right\} \in \Psi_{\omega}(X)$ converging to $X$, such that each $X_{n}$ is dense in itself, the space $Q\left(X ;\left\{X_{n}\right\}\right)$ is homeomorphic to the Menger universal curve $\mathcal{M}$.

Proof. Applying Properties1 1 and2, we see that $Q=Q\left(X ;\left\{X_{n}\right\}\right)$ is a curve. Next, $Q$ is locally connected and contains no local separating point by Property 3 Observe that the set $A=\left(C-\left\{a_{1}, b_{1}, a_{2}, b_{2}, \ldots\right\}\right) \times X$ is dense in $C \times X$ and each of its open subsets contains an uncountable family of mutually exclusive simple triods. Further, the mapping $q: C \times X \rightarrow Q$ restricted to $A$ is a homeomorphism. Therefore, each nonempty open subset of $Q$ also contains uncountably many mutually disjoint simple triods. Hence such a subset cannot be embedded into the plane by the Moore triodic theorem [5].

Finally, applying the well-known Anderson characterization theorem for the Menger curve [2], we obtain the conclusion.

Combining Propositions 7 and 4, the following general method of construction of upper semi-continuous (continuous) decompositions of the Menger curve is obtained. 
Let $X$ be a locally connected curve containing no free arc, and let $f: X \rightarrow Y$ be a continuous surjection such that each fiber $f^{-1}(y)$ is dense in itself. Next, take a sequence $\left\{Y_{n}\right\} \in \Psi_{\omega}(Y)$ such that sets $f^{-1}\left(Y_{n}\right)$ converge to $X$. Then mapping $g$ of Proposition 4 induces an upper semi-continuous decomposition of the topological Menger curve $Q\left(X ;\left\{f^{-1}\left(Y_{n}\right)\right\}\right)$ (Proposition 7) into sets homeomorphic to the respective fibers of mapping $f$ (Remark 5). Additionally,

(1) if $f$ is an open mapping, then this decomposition is continuous (Proposition (4); and

(2) if $Y$ is a curve containing no free arc and each $Y_{n}$ is dense in itself, then the quotient space $Q\left(Y ;\left\{Y_{n}\right\}\right)$ of this decomposition is again a topological Menger curve (Proposition 7 ).

In a previous paper [6] the author has proved that the Sierpiński universal plane curve $\mathcal{S}$ admits an open mapping $f$ onto itself with pseudo-arcs as all fibers. Let $X=Y=\mathcal{S}$ and take any sequence $\left\{Y_{n}\right\} \in \Psi_{\omega}(\mathcal{S})$ approximating $\mathcal{S}$ and composed of dense in themselves sets. Applying the above construction for this mapping $f$, we obtain the following main result of the paper.

Theorem 8. There exists a continuous decomposition of the Menger universal curve $\mathcal{M}$ into pseudo-arcs such that the quotient space is homeomorphic to $\mathcal{M}$.

Now, we briefly discuss the quotient spaces of continuous decompositions of $\mathcal{M}$ into pseudo-arcs. Let $\mathcal{C}_{\mathcal{M}}$ be the class of all such quotients. So $\mathcal{M} \in \mathcal{C}_{\mathcal{M}}$ by Theorem 8. Actually, an uncountable family of mutually non-homeomorphic, locally connected curves is contained in $\mathcal{C}_{\mathcal{M}}$. Indeed, they are obtained as $Q(\mathcal{S} ;\{\mathcal{Y} \backslash\})$ when arbitrary compacta approximating $\mathcal{S}$ and admitting isolated points are substituted for $Y_{n}$ in the last construction. In particular, if $Z$ is the union of two copies $M_{1}$ and $M_{2}$ of the Menger curve such that the set $M_{1} \cap M_{2}$ is embeddable into the plane and locally separates neither $M_{1}$, nor $M_{2}$ at each point, then $Z$ can be obtained as such $Q(\mathcal{S} ;\{\mathcal{Y} \backslash\})$. On the other hand, if all sets $Y_{n}$ are finite, we see that $Q(\mathcal{S} ;\{\mathcal{Y} \backslash\})$ is a curve containing no topological copy of $\mathcal{M}$. The generalizations mentioned in Remark 6 can also be used to produce some members of $\mathcal{C}_{\mathcal{M}}$.

However, not all locally connected continua belong to $\mathcal{C}_{\mathcal{M}}$. Indeed, applying [3, Th. 8, p.136, we see that each element of $\mathcal{C}_{\mathcal{M}}$ is one-dimensional. Moreover, we observe that for any $Z \in \mathcal{C}_{\mathcal{M}}$ each open subset of $Z$ contains a simple closed curve. To see this notice that, otherwise, the pre-image of a dendrite in $Z$ would be a subcontinuum of $\mathcal{M}$ with nonempty interior and trivial shape by $[7$, Th. 11 , an impossibility. Thus we have the following.

Proposition 9. Let $\mathcal{D}$ be a continuous decomposition of the Menger curve $\mathcal{M}$ into pseudo-arcs. Then the quotient space $\mathcal{M} / \mathcal{D}$ is a locally connected curve such that each nonempty, open subset of $\mathcal{M} / \mathcal{D}$ contains a simple closed curve.

So, the question of characterization of spaces in $\mathcal{C}_{\mathcal{M}}$ naturally appears. In particular we have the following problem.

Problem 1. Does there exist a locally connected curve $Z \notin \mathcal{C}_{\mathcal{M}}$ such that each open subset of $Z$ contains a simple closed curve? Does the Sierpinski curve $\mathcal{S}$ belong to $\mathcal{C}_{\mathcal{M}}$ ?

In the previous paper the author characterized all locally planar Peano continua admitting continuous decomposition into pseudo-arcs as those without local separating points, and it was proved that any Peano continuum having a local separating 
point has no continuous decomposition into acyclic curves ([6], Th.16 and Pr.15). We end the paper with the following general problem.

Problem 2. Characterize all Peano curves (continua) admitting continuous decomposition into pseudo-arcs (into acyclic curves).

\section{REFERENCES}

[1] R. D. Anderson, Open mappings of compact continua, Proc. Natl. Acad. Sci., U. S. A. 42(1956), 347-349. MR 17:1230h

[2] R. D. Anderson, One-dimensional continuous curves and homogeneity theorem, Ann. of Math. 68(1958), 1-16. MR 20:2676

[3] R. J. Daverman, Decompositions of manifolds, Academic Press, Inc. 1986. MR 88a:57001

[4] W. Lewis and J. J. Walsh, A continuous decomposition of the plane into pseudo-arcs, Houston J. Math. 4 (1978), 209-222. [MR 58:2750

[5] R. L. Moore, Concerning triods in the plane and the junction of points of plane continua, Proc. Natl. Acad. Sci., U. S. A. 14(1928), 85-88.

[6] J. R. Prajs, Continuous decompositions of Peano plane continua into pseudo-arcs, Fund. Math. 158 (1998), 23-40. CMP 99:01

[7] R. B. Sher, Realizing cell-like maps in euclidean space, Gen. Top. and Its Appl. 2 (1972), 75-89. MR 46:2683

[8] D. C. Wilson, Open mappings of the universal curve onto continuous curves, Trans. Amer. Math. Soc. 168 (1972), 497-515. MR 45:7682

Institute of Mathematics, Opole University, ul. Oleska 48, 45-052 Opole, Poland E-mail address: jrprajs@math.uni.opole.pl

Current address: Department of Mathematics and Statistics, Texas Tech University, Lubbock, Texas 79409-1042

E-mail address: prajs@math.ttu.edu 